\title{
Angioleiomyoma as a rare cause of a painful subcutaneous nodule in the leg: a case report
}

\author{
A.A. Younis', R.A. Hamed ${ }^{2}$, I.H. Abdulkareem² \\ ${ }^{1}$ Department of Medicine, Mosul College of Medicine, University of Mosul, Mosul, Iraq; \\ ${ }^{2}$ Radiology Department, Faruk Medical City, Sulaymaniah, Iraq
}

\begin{abstract}
Angioleiomyomas are benign tumors originating in the vascular smooth muscles. The tumor typically presents as a painful, solitary, small subcutaneous nodule. Herein, we have described a case report of chronic leg pain due to angioleiomyoma. We outline the clinical, radiological and histopathological features of this rare diagnosis for a painful nodule of extremity. Although rare, angioleiomyoma should be included in the differential diagnosis of chronic leg pain.
\end{abstract}

SUMMARY

Key words: Angioleiomyoma; vascular leiomyoma; leg pain; subcutaneous tumor.

\section{INTRODUCTION}

ngioleiomyoma or vascular leiomyoma is a rare, benign tumor arising from smooth muscle cells of the arterial or venous walls (1). This type of tumor can be found in the dermis, the subcutaneous fat or the deep fascia and it commonly occurs in the extremities, such as the lower leg (2). They can occur in any tissue containing smooth muscle including erector pili, smooth muscle wall of arteries, and genital tissues (vulva, nipple, and scrotum). They can also arise anywhere in the gastrointestinal tract and may present with obstruction, intussusception, or volvulus (3). Clinically, this tumor appears as a small $(<20 \mathrm{~mm})$, freely movable, subcutaneous nodule causing pain in approximately $60 \%$ of patients; it occurs more frequently in women than in men, and pregnancy may increase the severity of the pain (1).

Diagnosis of these lesions is frequently delayed because of their rare occurrence and due to the lack of awareness of this clinical condition. Since this lesion is rare, and the list of differential diagnoses for painful mobile subcutaneous mass is extensive, a case report and literature review of angioleiomyoma is presented.

\section{CASE REPORT}

A 32-year-old woman presented with 7 years' history of left leg pain. The patient's complaint started when she was pregnant with her first baby, suffering from severe left leg pain, located mainly in the anteromedial area of the lower half of the leg. The pain was often paroxysmal, particularly occurring after prolonged walking or during more intensive activity. After delivery the pain severity and frequency markedly decreased. The patient noticed that her pain markedly increased in the subsequent three pregnancies with less pain in between. However, during the last pregnancy 2 years ago, the pain became continuous, progressively increasing in severity, with paroxysms of excruciating pain, initiated even by a light touch or by exposure to wind or cold; with modest response to analgesics. She had no fever, involuntary weight loss or loss of energy. There was no history of trauma.

On physical examination, there was no skin discoloration, breakdown, or retraction, and the patient had no gross neurologic deficits of the lower extremity. The patient's examination was remarkable for a circumscribed mobile firm nodular mass,
Corresponding author: Ali Abdul Younis Department of Medicine, Mosul College of Medicine, University of Mosul, Mosul, Iraq E-mail: ali.younis7622@yahoo.com 


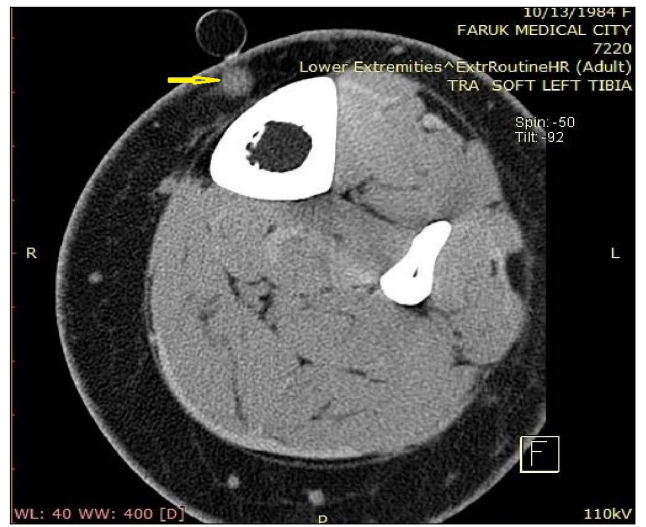

Figure 1 - CT scan of the left leg (axial) shows a small well defined round subcutaneous soft tissue mass, isodense to the skeletal muscles (arrow).

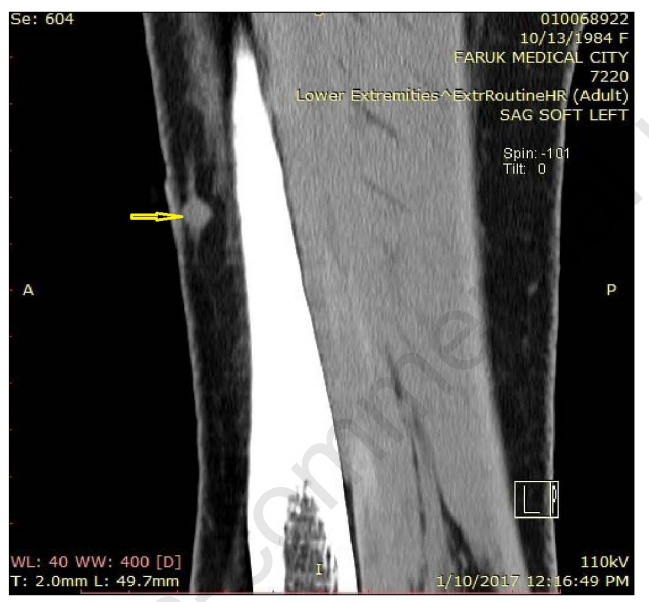

Figure 2 - CT scan of the left leg (sagittal) shows a small well-defined round subcutaneous soft tissue mass, isodense to the skeletal muscles (arrow).

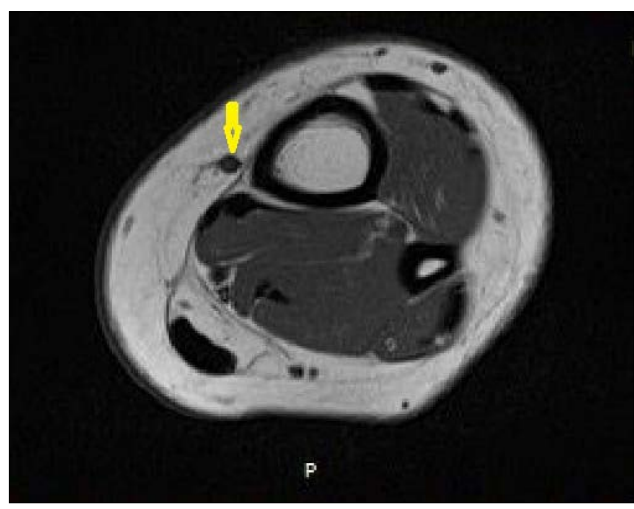

Figure 3 - Axial T1-weighted MR image of the left leg shows a small well-defined mass isointense to muscle (arrow). about $5 \mathrm{~mm}$ in diameter, located on the medial aspect of the mid-tibia. The overlying skin could be easily pinched from the lesion. The mass was exquisitely tender to palpation, with intense pain irradiating into the whole of the leg. The surrounding $5 \mathrm{~cm}$ was also tender, but to a less extent. Temperature and sensation of the overlying skin was normal.

The patient had undergone magnetic resonance imaging (MRI) of the left lower limb 1 year previously, which was reported as normal. Computed tomography (CT) scan had been ordered by an orthopedic surgeon, who initially suspected bone pathology, and it revealed small, well-defined, round subcutaneous soft tissue mass, isodense to the skeletal muscles, measuring $5 \mathrm{~mm}$ by 5 $\mathrm{mm}$, located in the anterior area of the left mid leg, with clear surrounding fat planes, and intact underlying bony cortex (Figures 1 and 2). On reviewing the MRI, the same mass was identified, eliciting an isointense signal from the skeletal muscles on $\mathrm{T} 1$ weighted image (Figure 3), and a slightly hyperintense signal on T2 weighted image, with thin dark signal rim (Figure 4), and it was closely related to the subjacent subcutaneous vein (Figure 5). Ultrasonography revealed a well-defined rounded hypoechoic mass lesion closely related to the adjacent blood vessel, and it was not vascular by color Doppler study.

A provisional diagnosis of angioleiomyoma was made. The patient underwent surgery with complete removal of the lesion



Figure 4 - Axial T2-weighted MR image of the left leg shows a small well-defined mass with slightly higher intensity than the muscles (arrow). 


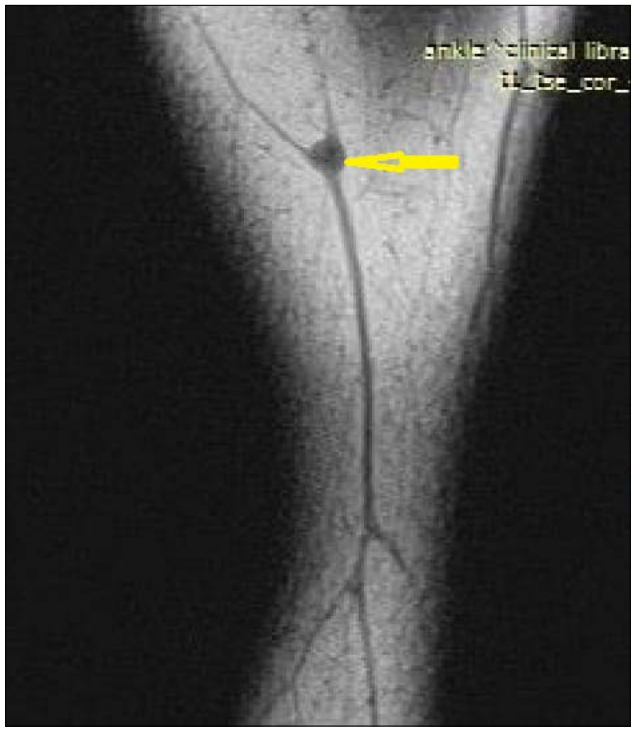

Figure 5 - Coronal T1-weighted MR image of the left leg shows a small well-defined mass closely related to the subjacent subcutaneous vein (arrow).

without complications. Macroscopic examination showed a whitish oval firm nodule that was solid, encapsulated, and grayish-white on the cut surface. Microscopic examination revealed a well-circumscribed spindle cells tumor arranged in fascicles that merged with smooth muscle bundles of the adjacent blood vessel. Histopathological examination was consistent with the diagnosis of angioleiomyoma (Figure 6). After surgery, the patient had complete relief of pain. There was no local recurrence at 6-month follow-up.

\section{DISCUSSION}

Angioleiomyomas are rare, benign, solitary tumors of smooth muscle cells originating in the muscular layer of the vessel walls (4). This lesion was first described by Stout in his comprehensive review, which was published in 1937 (5).

The condition is more common in females than in males (6). The tumors can affect individuals of any age, but they are more common in people between 30 and 60 years of age (1). Ninety per cent of these lesions occur in the extremities, with the

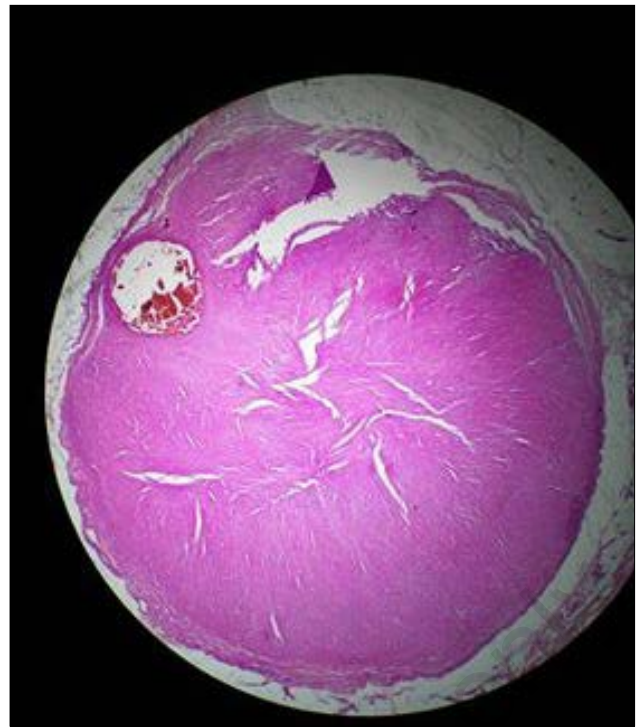

Figure 6 - Histological section shows well circumscribed spindle cells tumor arranged in fascicles that merge with smooth muscle bundles of the adjacent blood vessel (hematoxylin and eosin; $\times 40$ ).

head and trunk accounting for the remaining cases (1). Angioleiomyoma of the lower extremities are more frequent than upper extremities $(6,7)$. Among all the extremity angioleiomyomas, $50-70 \%$ are found in the lower extremity and it is twice as common in females. On the contrary, head, neck and upper extremity lesions are more common in males than females (1). The etiology is still unknown. In the series reported by Hachisuga et al. (5), 375 out of $562(66.7 \%)$ occurred in the lower extremity, $125(22.2 \%)$ in the upper extremity, 48 $(8.6 \%)$ on the head and $14(2.5 \%)$ on the trunk. Morimoto et al reviewed the clinicopathology of the vascular leiomyoma and they showed that $70 \%$ out of 241 cases were located below the knee (8).

Pain is the most striking clinical feature reported in about $60-75 \%$ of patients (1). The pain is often paroxysmal in nature, and has been described as sharp or pinching. It is initiated even by the slightest touch, or exposure to environmental stimuli such as change in temperature, exposure to wind, and other imperceptible stimuli. It can also be exacerbated by pregnancy or menses (4). Possible mecha- 
nisms explaining pain generation include contact of peripheral nerve fibers with the tumor (6), or active contracture of the tumor's smooth muscle elements resulting in local ischemia (9). Clinically, it consists of well-demarcated rounded masses, and most are less than $2 \mathrm{~cm}$ in diameter. This tumor, however, is rarely diagnosed before surgery, in spite of these characteristic clinical manifestations (4).

Differential diagnosis for painful subcutaneous extremity lesions includes, among others, angiolipomas, glomus tumors, ganglions, hemangiomas, and traumatic neuromas (1). Diagnosis depends on histopathological identification using staining techniques, such as Masson's trichrome, hematoxylin-eosin, Alcian blue, van Gieson, and periodic acid-Schiff (PAS). Immunohistochemical stains for vimentin, desmin and smooth muscle actin serve as an adjunct to histopathological identification of the angioleiomyoma (2).

Three histologic subtypes according to the predominant component have been described in literature:

1. capillary or solid angioleiomyomas which have rich smooth muscle cell stratification surrounding a few thin vascular channels;

2. venous angioleiomyomas which are characterized by more numerous and thicker vascular channels than are found in capillary angioleiomyomas;

3. cavernous angioleiomyomas which are dilated vascular channels surrounded by a thin layer of smooth muscle cells (10). Each histologic subtype has different clinical features with regard to location of the lesion and the gender of the patient, and the solid form is the most common subtype. The solid form is usually accompanied by pain, typically involves the lower extremity, and is 3 times as common in females $(4,11)$. The cavernous subtype is 4 times as common in males and accounts for a majority of the head and upper extremity lesions (12).

Ultrasonography and MRI are valuable in the diagnosis of vascular leiomyomas. The typical ultrasonographic appearance is a hypoechoic, homogeneous nodule with well-defined margins (13). The MRI finding of angioleiomyoma is a well-demarcated subcutaneous mass of isointense signal on T1-weighted image, heterogeneous mixed iso- or hyperintensity to muscles on T2-weighted images with homogeneous good enhancement, and an adjacent tortuous vascular structure are seen in the soft tissue of the extremities $(2,14)$. The treatment of angioleiomyoma by simple marginal excision is usually adequate (4).

\section{CONCLUSIONS}

In conclusion, angioleiomyoma is a rare soft tissue tumor presenting clinical symptoms which are typical, but non-specific. Angioleiomyoma should be considered a possible diagnosis when a well-demarcated subcutaneous mass of isointense signal on T1-weighted images, heterogeneous high signal intensity on T2-weighted images, and an adjacent tortuous vascular structure are seen in the extremities. Excision of the lesion permits histopathologic diagnosis and offers complete resolution of symptoms, as shown in this case.

\section{REEERRENCES}

1. Cigna E, Maruccia M, Malzone G, et al. A large vascular leiomyoma of the leg. J Ultrasound. 2012; 15: 121-3.

2. Sayit E, Sayit AT, Zan E, et al. Vascular leiomyoma of an extremity: report of two cases with MRI and histopathologic correlation. J Clin Orthop Trauma. 2014; 5: 110-4.

3. Willoughby AD, Schlussel AT, Freeman JH, Lin-Hurtubise K. Angioleiomyoma in a rare location: a case report. Hawai. J Med Public Health. 2013; 72: 45-8.

4. Cheung MH, Lui TH. Plantar heel pain due to vascular leiomyoma (angioleiomyoma). Foot Ankle Spec. 2012; 5: 321-3.

5. Hachisuga T, Hashimoto H, Enjoji M. Angioleiomyoma: a clinicopathologic reappraisal of 562 cases. Cancer. 1984; 54: 126-30.

6. Boutayeb F, Ibrahimi AE, Chraibi F, Znati K. Leiomyoma in an index finger: report of case and review of literature. Hand. 2008; 3: 210-1.

7. Kulkarini AR, Haase SC, Chung KC. Leiomyoma of the hand. Hand. 2009; 4: 145-9.

8. Morimoto N. Angiomyoma: a clinicopathologic study. Med J Kagoshima Univ. 1973; 24 : 663-83. 
9. Woo KS, Kim AH, Kim HS, Cho PD. Clinical experience with treatment of angioleiomyoma. Arch Plast Surg. 2014; 41: 374-8.

10. Gajanthodi S, Rai R, Chaudhry RK. Vascular leiomyoma of foot. J Clin Diagn Res 2013; 7: 571-2.

11. Ogura K, Goto T, Nemoto T. Painless giant angioleiomyoma in the subfascia of the lower leg. J Foot Ankle Surg. 2012; 51: 99-102.

12. DiCaprio MR, Jokl P. Vascular leiomyoma presenting as medial joint line pain of the knee. Arthroscopy. 2003; 19: E24.

13. Yamashita K, Shimmura H, Tokiwa S, Kato S. Painless vascular leiomyoma found after incision of the vaginal wall: a case report. Urol Case Rep. 2018; 17: 19-21.

14. Yoo HJ, Choi JA, Chung JH, et al. Angioleiomyoma in soft tissue of extremities: MRI findings. AJR Am J Roentgenol. 2009; 192: 291-4. 